\title{
Application of Wavelet Transform Analysis to ADCs Harmonics Distortion
}

\author{
Emad A. Awada ${ }^{1}$ \& Mohammad H. Alomari ${ }^{1}$ \\ ${ }^{1}$ School of Electrical and Computer Engineering, Applied Science University, Amman, Jordan \\ Correspondence: Emad A. Awada, School of Electrical and Computer Engineering, Applied Science University, \\ Amman, Jordan. P. O. Box 166, Amman 11931 Jordan. Tel: 962-65-60-9999 ext. 1094. E-mail: \\ e_awada@asu.edu.jo
}

Received: March 20, 2013 Accepted: June 24, 2013 Online Published: July 8, 2013

doi:10.5539/cis.v6n3p118 URL: http://dx.doi.org/10.5539/cis.v6n3p118

\begin{abstract}
This paper presents a new method of detecting Analog to Digital Converter harmonic distortion. The new method is based on Wavelet multi-resolution process to identify instantaneous harmonic components. In classical testing, Fourier transform algorithm was long adopted to estimate Total Harmonic Distortion by obtaining signal power spectrum. While the conventional method of Fourier transform tend to be complicated and lengthy, the new investigated algorithms of Wavelet transform has shown less computations process and instantaneous testing of ADCs harmonic distortions. By shorten testing times and reduced computation complexity, Wavelet transform can be particularly appropriate for developing ADCs low-cost, and fast testing procedure.
\end{abstract}

Keywords: Total Harmonic Distortion (THD), Discrete Wavelet Transforms (DWT), Mixed Signal Analog-toDigital Converters (ADCs)

\section{Introduction}

Mixed signal devices provide the ability of transforming information across Analog and/or Digital domains as desired (Burns \& Roberts, 2004; Akujuobi et al., 2007). Therefore, in choosing the right data acquisition board, signal conversion accuracy can be one of the most important factors. For proper system performance, ADCs digital output must be close to analog input information signal. At most basic level, testing of mixed signal devices would seem simple matter; however, testing is extremely expensive, and time consuming as in (Akujuobi et al., 2007; Yamaguchi \& Soma, 1997; Marshall \& Akujuobi, 2002; Awada et al., 2010) for both static and dynamic parameter characterizations. Dynamic parameters have been always based on FT computation to provide specific information that shows the effect of noise and signal distortions. Noise and signal distortions can be related to the effect of external and internal sources (Akujuobi et al., 2007; Yamaguchi \& Soma, 1997; Awada \& Akujuobi, 2010). For instant, in ADC's operation, quantization errors cause nonlinearity effect that results into spectral frequencies other than the input frequency appear as random noise spread across the frequency spectrum. Such a distortion results in harmonic distortions and higher noise floor. Therefore, THD measurements can be one of the main dynamic error characteristics of ADCs testing. Classical testing of FT has shown lengthy and complicated process due to the large number of data samples (Akujuobi et al., 2007; Yamaguchi \& Soma, 1997; Marshall \& Akujuobi, 2002; Awada et al., 2010) in addition to failure in cases of non-stationary signal (Awada et al., 2010; Tuntisak, 2007; Qingyang \& Zhe, 2012; Sangeethapriya, 2005). As a result, significant research has been done in the area of testing ADCs to improve testing techniques. While some focused on improving classical methods such as Fourier transform (Cherubal \& Chatterjee, 2003; Wen-Ta \& Yi-Zhen, 2008; Serra \& DaSilva, 2005), others have concentrated on new testing algorithms such as Wavelet transform (Akujuobi et al., 2007; Yamaguchi \& Soma, 1997; Marshall \& Akujuobi, 2002; Awada et al., 2010; Akujuobi \& Hu, 2003; Gandelli \& Ragaini, 1996). Through simulation process, Wavelets have shown improvements and satisfactory results in term of accuracy and simplicity in testing for instantaneous THD. With the focus on shortening testing process, reducing sample size, and simplifying testing process, Wavelet computations of ADCs THD was very close to conventional methods of FT.

\section{Therotical Background}

In quantifying Mix signal converters dynamic performance, noise and signal distortion are determined based on FT analysis where fundamental signal characteristic can be determine via frequency spectrum (Balestrieri \& 
Rapuano, 2007; Bellan et al., 1998). To provides a quantitative measurement of THD, as defined in IEEE Std. 1241 , THD is the ratio of harmonics power summation to the fundamental frequency power

$$
T H D=\frac{P_{2}+P_{3}+P_{4}+\ldots+P_{\infty}}{P_{1}}
$$

Generally speaking, all spectrum components, $\mathrm{n}$ multiple of the fundamental frequency, are considered harmonics with effect determined by THD. Yet, this method of testing THD is extremely sensitive to errors caused by noise, especially with the fact FFT test depend on noise summation including quantization and even very small noises caused by internal component of IC board (Burns \& Roberts, 2004; Yamaguchi \& Soma, 1997).

\section{Wavelet Transform}

The classical method of FT performs very well in analyzing periodic stationary signal. However, it is not the case for non-stationary signal or even a sudden changes (voltage dips) occurs in the waveform (Sangeethapriya, 2005; Bingyi \& Haian, 2007). Meanwhile, Wavelet transforms powerful computation and mathematical tools are used in various fields of signal processing (Riouel \& Vetterli, 1991; Silverman, 2000; Oliver, Shantha, \& Sadasivam, 2005). Wavelet properties of fully scalable window, based on multi-resolutions process, allow pinpointing signal components. These special properties of dilation and translation enable Wavelets to create different scale and shifted functions of a signal to conclude all components for every position (Tuntisak, 2007; Qingyang \& Zhe, 2012). In other words, unlike the Fourier transform, Wavelet scalable window shift a signal in time domain, (rescaling -expand or compress a signal), and produces flexible windows for analysis. As a result, Wavelet finer and large scaling, permit all information of the signal (the big picture), while small scales shows signal details by zooming into the signal components. In (Mallat, 1999), wavelet ( $\Psi$ ) is a function of zero average as, i.e.

$$
\int_{-\infty}^{\infty} \psi(t) d t=0
$$

where $(\Psi)$ is base function known as mother wavelet used to drive wavelet transformation function through dilation (s) and translation $(\mathrm{u})$

$$
\psi_{u, s}(t)=\frac{1}{\sqrt{s}} \psi\left(\frac{t-u}{s}\right)
$$

And since Wavelet merely performs a convolution operation with a given signal, Wavelet transform of continuous signal $\mathrm{x}(\mathrm{t})$ can be illustrated as

$$
W x(u, s)=\int_{-\infty}^{\infty} X(t) \frac{1}{\sqrt{s}} \psi^{*}\left(\frac{t-u}{s}\right)
$$

However, dealing with discrete signal, Discrete Wavelet Transform (DWT) is better fit for this analysis of ADCs. A large selection of DWT families is available with predetermined low-pass and high-pass filter banks for each particular mother Wavelet (Silverman, 2000; Oliver, Shantha, \& Sadasivam, 2005). However, the selection of a particular Mother Wavelet should based on Wavelet shape, characteristics, matching signal, orthogonality, and filter linearity (Mallat, 1999; Shantha \& Sadasivam, 2005; Peng \& Dong, 2005). DWT can be classified in two major categories: 1. Orthogonal Wavelets-Haar, Daubechies (dbxx), Coiflets (coifx) etc. Orthogonal wavelets (not linear phase filters) are suitable for applications such as signal and image compression and de-noising (Bellan et al., 1998); 2. Biorthogonal wavelets_-FBI, and Biorthogonal (biorx_x). Such types of wavelets are linear-phase filters that maintain a constant time delay for different frequencies and are necessary in signal extraction applications, such as peak detection (Hawwar \& Reza, 2000).

In this work of testing ADCs, sine waveform was used as stimulus signal (Figure 1a). The selection of Wavelet was based on stimulus signal to allow best analysis; such as, Daubechies and Coiflets Wavelet (Figure 1b). In addition, Biorthogonal Wavelet (Figure 1c) was used as well to demonstrate analysis comparences among all algorithms. 


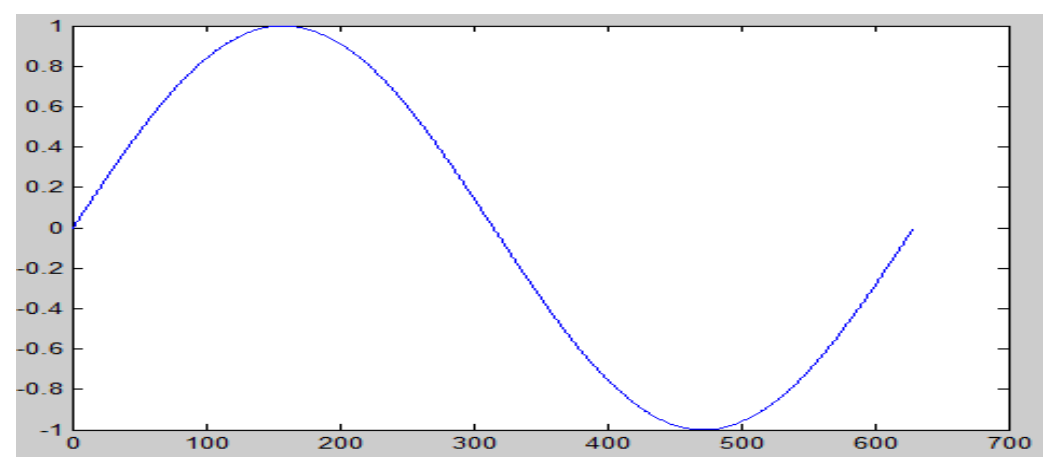

Figures 1a. Sine waveform

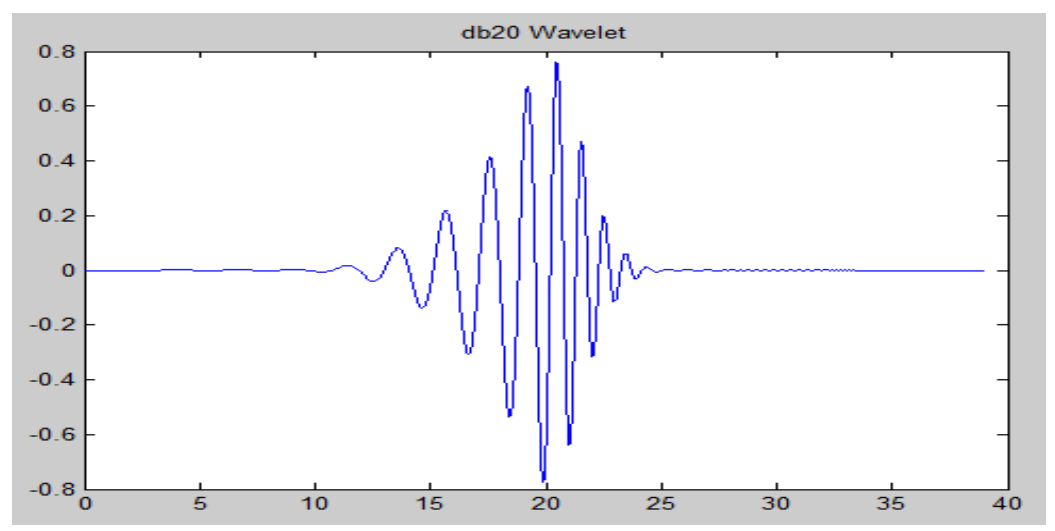

Figures 1b. Daubechie wavelet

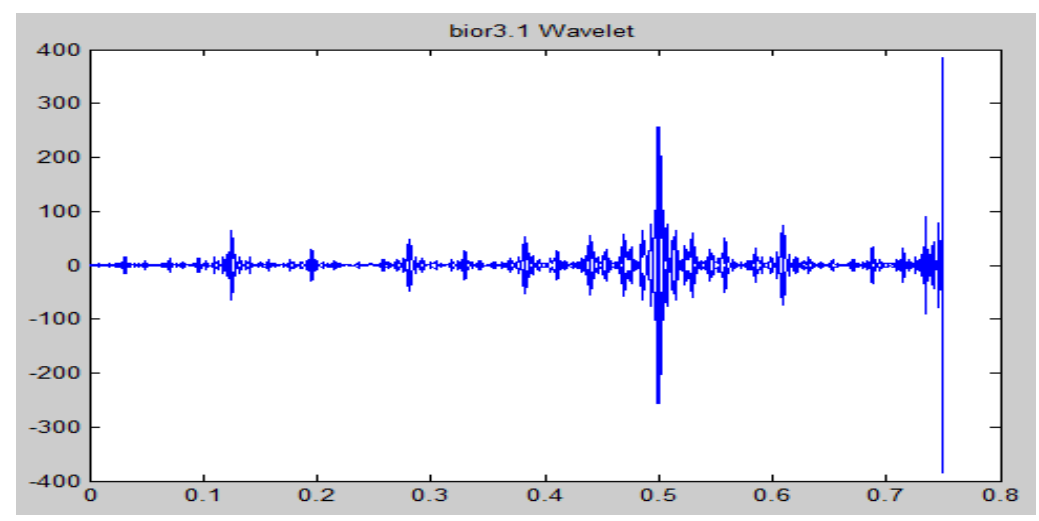

Figures 1c. Biorthogonal wavelet

\section{Wavelet Analysis and THD}

The benefit of discrete Wavelet is applied by considering sub-band decomposition using digital filter bank as low and high pass. While high pass provide detail approximations, low pass filter approximate the signal. As a result, a given bandwidth will be separated to obtain detail components of each multi-resolution level shown in Figure 2 .

Theoretically, by applying clean perfect sine wave, stimulus signal parameters such as (amplitude, frequency, phase, dc offset, etc) can be determined and the deviation of ADC output signal from the ideal input signal is an effect of ADC performance. For an ideal ADC, the deviation is negligible (zero). However, in reality, ADC digital output $\bar{x}(t)$ contains errors due to noise, distortion error, heat, i.e. 


$$
\widehat{X}[n]=x(t)+e
$$

where $x(t)$ is Original input signal, and $e$ is error-value.

By capturing ADC output $(\widehat{X}[n])$ and Appling DWT algorithms, the output signal will be decomposed into combination of instantaneous low frequency components (approximation coefficients $\mathrm{cAj}$ ) and high frequency (detail coefficients cDj) at sub-band (j). In (Tuntisak, 2007; Qingyang \& Zhe, 2012; Bingyi \& Haian, 2007), distortion rate associated with harmonics is estimated from relevant approximation and detail coefficient of each sub-band. In which the coefficients of low frequency $\left(\mathrm{cA}_{\mathrm{j}}\right)$ are considered as fundamental components of each sub-band and the high frequency coefficients $\left(\mathrm{cD}_{\mathrm{j}}\right)$ extracted in each level of decomposition as illustrated in Figure 2 .

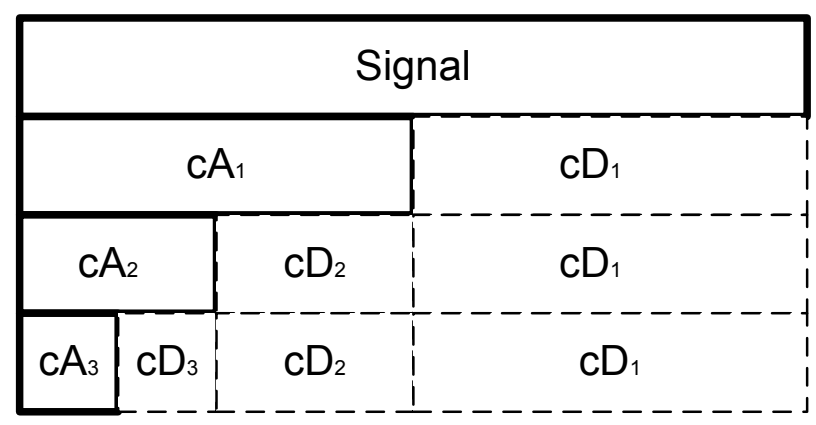

Figure 2. Multi-resolution decomposition

Therefore, the estimation of THD can be obtained by the summation of harmonic components in the detail approximation cDj. By (Tuntisak, 2007; Sangeethapriya, 2005), harmonics distortion given by RMS of detail approximation coefficients in each sub band

$$
R M S=\sqrt{\frac{1}{N_{j}} \sum_{n}\left[c D_{j}(n)\right]^{2}}
$$

By summing all harmonic components in multi sub-band, THD can be express as

$$
T H D=\sqrt{\sum_{j}\left(\frac{1}{N_{j}}\right) \sum_{n}\left[c D_{j}(n)\right]^{2}}
$$

where $\mathrm{j}$ is level of decomposition, and $\mathrm{N}$ is number of coefficients.

\section{Simulation Results}

A simulated waveform was used for testing validation of harmonics detection algorithms in both FTT and DWT. Random noise was added for testing accuracy as shown in Figure 3.

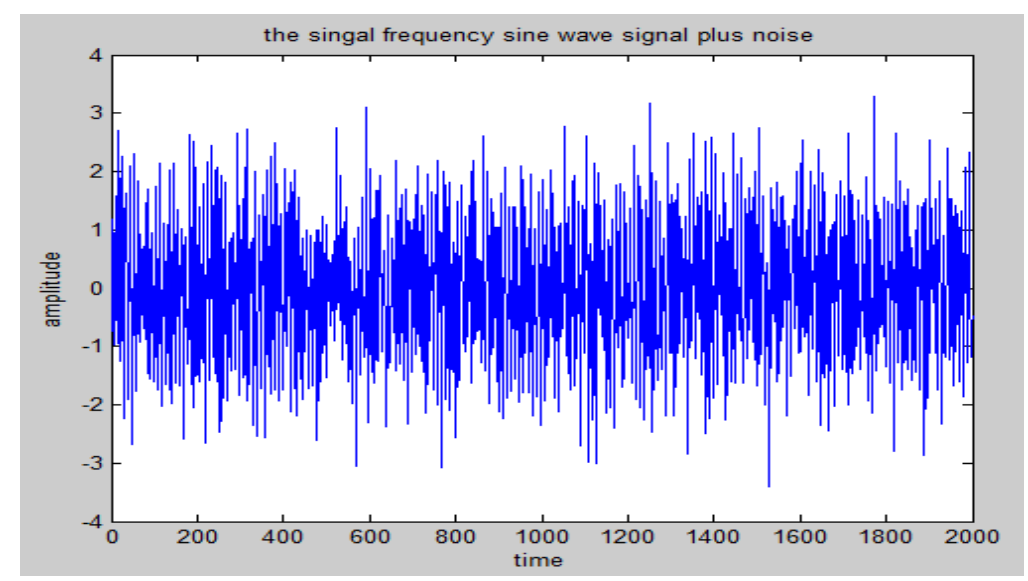

Figure 3. Distorted waveform under test 
Matlab program was used for this simulation utilizing fundamental frequencies of $100 \mathrm{~Hz}, 150 \mathrm{~Hz}$, and $200 \mathrm{~Hz}$, ideal 10 bit ADC, and sampling frequency fs $(1000 \mathrm{~Hz})$ to satisfy the Nyquist theorem. By using FFT, waveform was transferred into frequency spectrum in Figure 4.

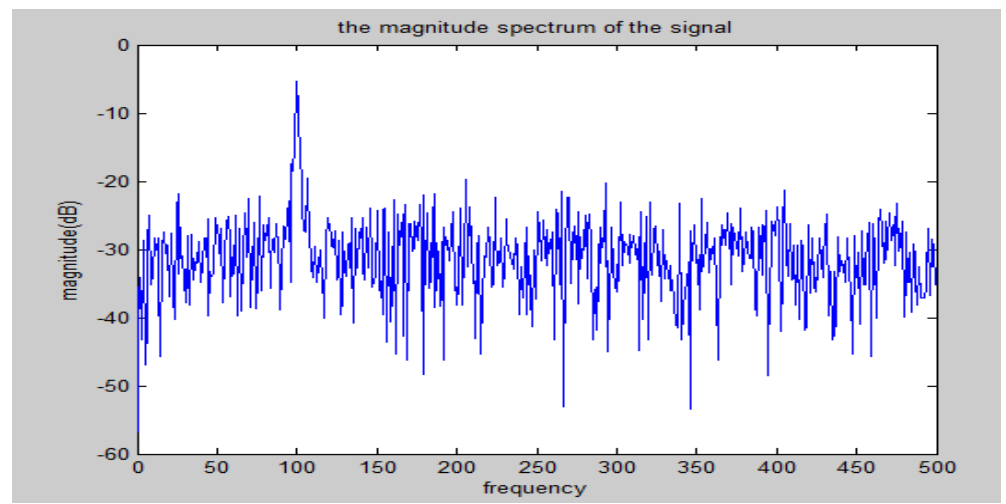

Figure 4. Frequency spectrum at $100 \mathrm{~Hz}$

Each harmonics component and total distortion of the waveform were calculated first by obtaining the power spectrum and compute the ratio of total harmonics power to fundamental signal power as in Figure 5.

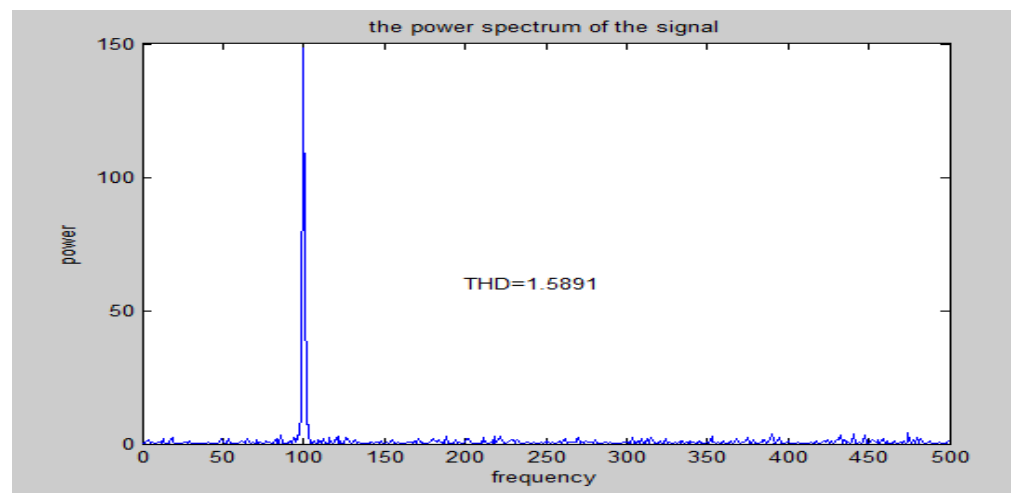

Figure 5. Power spectrum

Meanwhile, DWT algorithms were applied to compute instantaneous THD. The original distorted waveform was decomposed into sub-bands using different type of discrete wavelet such as; db14, db20, Coilf1, and Bior3.1 as discussed in Section 3. For instance, discrete wavelet db20 was applied in five -decomposition levels to original distorted waveform, shown in Figure 6, and harmonic components in each detail level were added as in section 4.

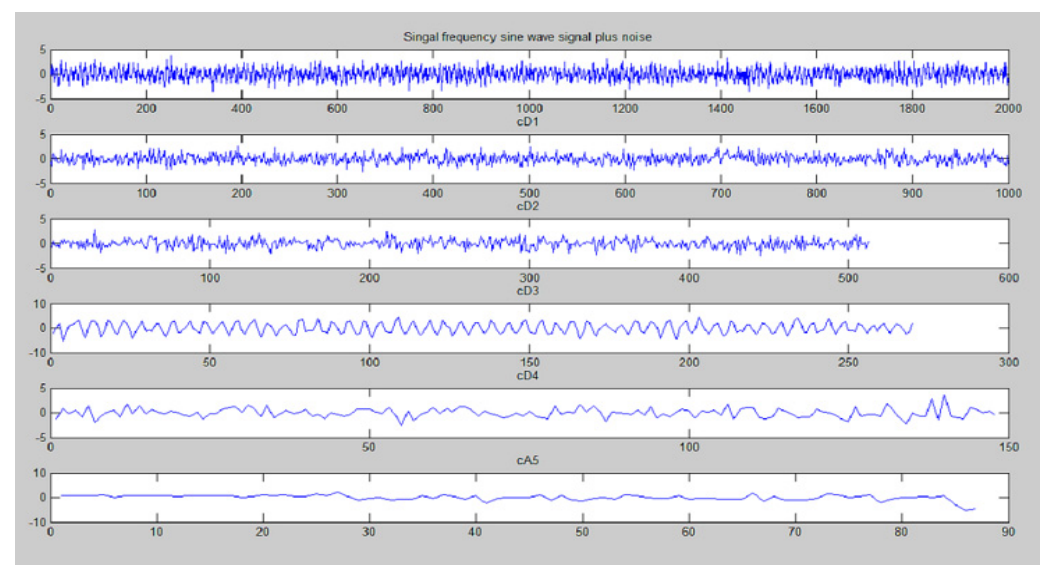

Figure 6. Wavelet decomposition using db20 
To validate experiment results, testing was performed on several frequencies and types of DWT. Results were validated with classical testing of FFT as shown in Table 1, and Figure 7.

Table 1. THD at various frequencies and analysis techniques

\begin{tabular}{cccccc}
\hline Frequency & FFT & Db14 & Db20 & Coef1 & Bior3-1 \\
\hline $100 \mathrm{~Hz}$ & $\mathbf{1 . 5 9}$ & $\mathbf{1 . 3 1}$ & $\mathbf{1 . 3 1}$ & $\mathbf{1 . 1 7}$ & $\mathbf{4 . 7 2}$ \\
$150 \mathrm{~Hz}$ & $\mathbf{1 . 9 0}$ & $\mathbf{1 . 4 0}$ & $\mathbf{1 . 9 0}$ & $\mathbf{1 . 4 1}$ & $\mathbf{4 . 9 3}$ \\
$200 \mathrm{~Hz}$ & $\mathbf{1 . 9 5}$ & $\mathbf{1 . 3 3}$ & $\mathbf{1 . 8 6}$ & $\mathbf{1 . 4 6}$ & $\mathbf{4 . 8 8}$ \\
\hline
\end{tabular}

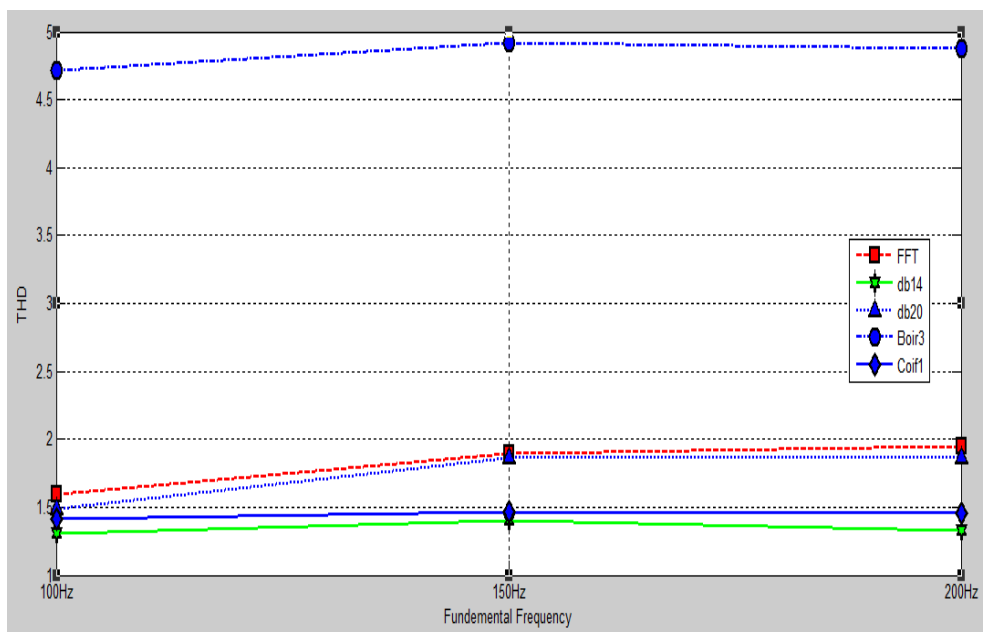

Figure 7. THD values in Table 1

As seen from Table 1 and Figure 7, db20 was best fit for this type of signal processing based on the matching signal characteristics for this application of testing using sinusoid waveform. Also, while db14 and Coif have close results to FFT analysis, Biorthogonal analysis was significantly off.

\section{Conclusion}

In this paper, wavelet transform algorithm was applied to analyze harmonics components in mixed signal converters. As shown, it is clear that wavelet can provide an alternative method of testing and estimation for THD. Using the special properties of multi-resolution, instantaneous harmonic distortion can be estimated with less data. Such a benefit of wavelet algorithms allows to decrease number of data samples, expedite testing process, reduce testing cost, and enhance analysis results. From this simulation and other works had been done in this field, Wavelet can be practically suitable testing tools, especially with the growing demands for built in self-test impeded algorithms, and self-calibration schemes.

\section{Acknowledgements}

The authors are grateful to ASU, Amman - Jordan, for the financial support grated to cover the publication fee of this paper research articles.

\section{References}

Akujuobi, C. M., Awada, E., Sadiku, M., \& Ali, W. (2007). Wavelet-Based Differential Nonlinearity Testing of Mixed Signal System ADCs. IEEE Proceeding - Southeast Conference., 76-81.

Akujuobi, C. M., \& Hu, L. (2003). Implementation of the wavelet transform-based technique for static testing of mixed signal system. IASTED Conference, 56-59.

Awada, E., Akujuobi, C. M., \& Sadiku, M. (2010). A Reduced Code Linearity Test for DAC Using Wavelet Analysi. International Journal of Engineering Research \& Innovation (IJERI), 2, 69-76.

Awada, E., \& Akujuobi, C. M. (2010). DWT Testing of DAC Effective Number of Bits. IASTED Conference on Circuits and Systems, 1-6. http://dx.doi.org/ 10.2316/P.2010.712-001 
Balestrieri, E., \& Rapuano, S. (2007). Defining DAC performance in the frequency domain Measurement. Journal: Measurement, 40, 463-472. http://dx.doi.org/10.1016/j.measurement.2006.12.004

Bellan, D., Brandolini, A., \& Gandelli, A. (1998). ADC nonlinearities and harmonic distortion in FFT test. IEEE Conference on Instrumentation and Measurement Technology, 2, 1233-1238.

Bingyi, Z., \& Haian, C. (2007). Application of Wavelet Multi-Resolution Analysis to Harmonics DetectionBased on MATLAB in Power System. IEEE International Conference on Automation and Logistics, 137-143.

Burns, M., \& Roberts, G. W. (2004). ADC Testing. In An Introduction to Mixed-Signal IC Test and Measurement. (pp. 447-479). Oxford, New York, NY: Oxford University Press.

Cherubal, S., \& Chatterjee, A. (2003). Optimal linearity testing of analog-to-digital converters using a linearmodel. IEEE Transactions on Circuits and Systems I: Fundamental Theory and Applications, 50, 317-327. http://dx.doi.org/10.1109/TCSI.2003.809775

Gandelli, A., \& Ragaini, E. (1996). ADC transfer function analysis by means of a mixed wavelet-walsh transform. IEEE Conference on Instrumentation and Measurement technology, 2, 1314-1318.

Hawwar, Y. M., \& Reza, A. M. (2000). Nonlinear filtering in the wavelet transform domain. IEEE International Conference, 3, 266-269.

Mallat, G. S. (1999). Introduction to a Transient World. In Wavelet tour of signal processing (pp. 24-57). United Kingdom, UK: Academic Press. http://dx.doi.org/10.1016/B978-012466606-1/50003-9

Marshall, R. O., \& Akujuobi, C. M. (2002). On The Use of Wavelet Transform in Testing for The DNL of ADCs. IEEE Midwest Symposium on Circuits and Systems, 2, 25-28.

Oliver, J., Shantha, S. K., \& Sadasivam, V. (2005). Wavelets for improving spectral efficiency in a digital communication system. Computational Intelligence and Multimedia Applications, 198-203.

Peng; L., \& dong, J. (2005). Characteristic Analysis and Selection of Wavelets Applicable for Ultra-High-Speed Protection. IEEE Conference on Transmission and Distribution, 1-5.

Qingyang, L., \& Zhe, S. (2012). Method of Harmonic Detection Based On the Wavelet Transform. International Conference on Information and Computer Applications (ICICA), 24, 213-217.

Riouel, O., \& Vetterli, M. (1991). Wavelet and signal processing. IEEE SP Mag., 8, 14-38. http://dx.doi.org/10.1109/79.91217

Sangeethapriya, S. (2005). Harmonic Detection Using Wavelet Transform. IEEE Proceeding Conference Neural Networks, 4, 2228-2231.

Serra, C. A., \& DaSilva, M. F. (2005). Combined spectral and histogram analysis for fast ADC testing. IEEE Transactions on Instrumentation and Measurement, 54, 1617-1623. http://dx.doi.org/10.1109/TIM.2005.851057

Silverman, B. (2000). Wavelets in statics: beyond the standard assumptions. In Wavelet: The key to intermittent information (pp. 71-86). Oxford, New York, NY: Oxford University Press.

Tuntisak, S. (2007). Harmonic Detection in Distribution Systems Using Wavelet Transform and Support Vector Machine. IEEE Lausanne, Conference on Power Tech., 1540-1545. http://dx.doi.org/10.1109/PCT.2007.4538544

Wen-Ta, L., \& Yi-Zhen, L. (2008). A high precision ramp generator for low cost ADC test. IEEE 9th International Conferen on Solid-State and Integrated-Circuit Technology, 2103-2106.

Yamaguchi, T., \& Soma, M. (1997). Dynamic Testing of ADCs Using Wavelet Transform. IEEE International Test Conference, 379-388.

\section{Copyrights}

Copyright for this article is retained by the author(s), with first publication rights granted to the journal.

This is an open-access article distributed under the terms and conditions of the Creative Commons Attribution license (http://creativecommons.org/licenses/by/3.0/). 